\title{
Kaluza-Klein rotating multi-black hole configurations with electromagnetic field in Einstein-Maxwell-dilaton gravity
}

\author{
Stoytcho S. Yazadjiev * \\ Department of Theoretical Physics, Faculty of Physics, Sofia University, \\ 5 James Bourchier Boulevard, Sofia 1164, Bulgaria
}

\begin{abstract}
We present a new solution in 5D Einstein-Maxwell-dilaton gravity describing an equilibrium configuration of extremal rotating black holes with lens space horizon topologies. The basic properties of the solution are investigated and the basic physical quantities are calculated. It is shown that the black hole horizons are superconducting in the sense that they expel the magnetic flux lines.
\end{abstract}

In the last decade the higher dimensional gravity has attracted a lot of interest and it is now a well established area of the modern theoretical and mathematical physics. The spacetimes with compact extra dimensions (Kaluza-Klein spacetimes) take a special place since they are much more realistic than the asymptotically flat spacetimes. During the last years Kaluza-Klein black holes were extensively studied and many exact solutions were found but a lot remains to be done in this direction since the spectrum of black hole solutions in the Kaluza-Klein case is much richer than in the asymptotically flat case. For recent review on the subject we refer the reader to [1].

In the present paper we deal with multi-black hole Kaluza-Klein spacetimes. Such multi-black hole configurations are very interesting since they provide us with valuable insight into the black hole theory in spacetimes with compact extra dimensions and especially into the black hole interactions in the strong field regime. Very recently a Kaluza-Klein rotating vacuum multi-black hole solution in five dimensions was studied in [2]. The authors show that the previously found solution of [3] can be interpreted as an equilibrium configuration of extremal rotating black holes held apart by the repulsive spin-spin interaction. The solution was generalized to the case of 5D Einstein-Maxwell gravity and 5D minimal supergravity in [4].

The purpose of the present paper is to present a new solution describing an equilibrium configuration of extremal rotating black holes with selfgravitating electromagnetic

*E-mail: yazad@phys.uni-sofia.bg 
field in the 5D Einstein-Maxwell-dilaton gravity and to study some of its basic properties. Our solution differs from those in [4] in that it is not charged and the magnetic fluxes through the horizons are zero.

We consider the five dimensional Einstein-Maxwell-dilaton (EMD) gravity given by the action

$$
S=\frac{1}{16 \pi} \int d^{4} x \sqrt{-g}\left[\mathcal{R}-2 g^{\mu \nu} \partial_{\mu} \varphi \partial_{\nu} \varphi-e^{-2 \alpha \varphi} F^{\mu \nu} F_{\mu \nu}\right]
$$

where $\mathcal{R}$ is the Ricci scalar curvature with respect to the spacetime metric $g_{\mu \nu}, F_{\mu \nu}$ is the Maxwell tensor, $\varphi$ is the dilaton field and $\alpha$ is the dilaton coupling constant. The field EMD equations are obtained by varying the action:

$$
\begin{aligned}
& \mathcal{R}_{\mu \nu}=2 \nabla_{\mu} \varphi \nabla_{\nu} \varphi+2 e^{-2 \alpha \varphi}\left[F_{\mu \sigma} F_{\nu}^{\sigma}-\frac{g_{\mu \nu}}{6} F^{\rho \sigma} F_{\rho \sigma}\right] \\
& \nabla_{\mu} \nabla^{\mu} \varphi=-\frac{\alpha}{2} e^{-2 \alpha \varphi} F^{\rho \sigma} F_{\rho \sigma} \\
& \nabla_{\mu}\left[e^{-2 \alpha \varphi} F^{\mu \nu}\right]=0 .
\end{aligned}
$$

In the present paper we will consider the case $\alpha=\sqrt{8 / 3}$. For this value of the dilaton coupling parameter we have found the following solution

$$
\begin{aligned}
& d s^{2}=V^{-1 / 3}\left\{-H^{-2} d t^{2}+\left[\sqrt{2}\left(H^{-1}-1\right) d t+L d \psi+\sqrt{2} \cosh \gamma \hat{W}\right]^{2}\right\} \\
& +V^{2 / 3} H^{2}\left[d x^{2}+d y^{2}+d z^{2}\right] \\
& A_{\mu} d x^{\mu}=-\frac{\sinh \gamma}{2}\left(H^{-1}-1\right)^{2} d t-\frac{\sinh \gamma}{\sqrt{2}}\left(H^{-1}-1\right) L d \psi-\cosh \gamma \sinh \gamma H^{-1} \hat{W},(6) \\
& e^{\sqrt{2 / 3} \varphi}=V^{1 / 3}
\end{aligned}
$$

where the metric function $V$ and the 1 -form $\hat{W}$ are given by

$$
\begin{aligned}
& V=\cosh ^{2} \gamma-H^{-2} \sinh ^{2} \gamma \\
& \hat{W}=\sum_{i} m_{i} \frac{z-z_{i}}{\left|R-R_{i}\right|} \frac{\left(x-x_{i}\right) d y-\left(y-y_{i}\right) d x}{\left(x-x_{i}\right)^{2}+\left(y-y_{i}\right)^{2}}
\end{aligned}
$$

and $H$ is a harmonic function on the three dimensional flat space explicitly given by

$$
H=1+\sum_{i} \frac{m_{i}}{\left|R-R_{i}\right|}
$$

with point sources located at $R_{i}=\left(x_{i}, y_{i}, z_{i}\right)$. The 1 -form $\hat{W}$ and the harmonic function $H$ satisfy the following equation on the three dimensional flat space 


$$
\nabla \times \hat{W}=\nabla H
$$

The parameters $\gamma, L m_{i}$ run in the ranges $-\infty<\gamma<\infty, L>0$ and $m_{i} \geq 0$. In the case $\gamma=0$ our solution reduces to the vacuum solution of [2]. In the general case the above metric possesses only two Killing fields $\frac{\partial}{\partial t}$ and $\frac{\partial}{\partial \psi}$.

Without loss of generality we will consider the case of two black holes with $R_{1}=$ $(0,0,0)$ and $R_{2}=(0,0, a)$ where $a>0$. In this case the flat three metric, the harmonic function $H$ and the 1 -form $\hat{W}$ are given by

$$
\begin{aligned}
& d s_{3}^{2}=d x^{2}+d y^{2}+d z^{2}=d R^{2}+R^{2}\left(d \theta^{2}+\sin ^{2} \theta d \phi^{2}\right), \\
& H=1+\frac{m_{1}}{R}+\frac{m_{2}}{\sqrt{R^{2}+a^{2}-2 R a \cos \theta}}, \\
& \hat{W}=\left(m_{1} \cos \theta+m_{2} \frac{R \cos \theta-a}{\sqrt{R^{2}+a^{2}-2 a R \cos \theta}}\right) d \phi .
\end{aligned}
$$

In addition to the Killing fields $\frac{\partial}{\partial t}$ and $\frac{\partial}{\partial \psi}$, in the case under consideration there is one more Killing field $\frac{\partial}{\partial \phi}$. The coordinates satisfy $-\infty<t<\infty, 0<R<\infty$, $0 \leq \theta \leq \pi, 0 \leq \phi<2 \pi$ and $0 \leq \psi<2 \pi$.

Using a standard approach one can show that the point sources $R=R_{1}$ and $R=R_{2}$ correspond to smooth horizons for the metric. The coordinates we use to write solutions are actually singular on the horizons and therefore we should introduce new coordinates that cover the horizons too. We will do so only for the horizon at $R=0$ since the other horizon can be treated analogously. Near the horizon we introduce the new coordinates $v$ and $\tilde{\psi}$ given by

$$
\begin{aligned}
& d v=d t-\cosh \gamma m_{1} d\left(\frac{m_{1}}{R}\right) \\
& d \tilde{\psi}=d \psi-\frac{\sqrt{2}}{L} d t-\frac{\sqrt{2} \cosh \gamma m_{1}}{L} \frac{d R}{R}-\frac{\sqrt{2} \cosh \gamma m_{2}}{L} d \phi .
\end{aligned}
$$

In the new coordinates the metric takes the form

$$
\begin{array}{r}
d s^{2}=\cosh ^{-2 / 3} \gamma\left\{\frac{R^{2}}{m_{1}^{2}} d v^{2}+2 \cosh \gamma d v d R\right. \\
+m_{1}^{2} \cosh ^{2} \gamma\left[d \Omega^{2}+2\left(\frac{L}{\sqrt{2} m_{1} \cosh \gamma} d \tilde{\psi}+\cos \theta d \phi\right)^{2}\right] \\
\left.+4 \cosh \gamma R d v\left(\frac{L}{\sqrt{2} m_{1} \cosh \gamma} d \tilde{\psi}+\cos \theta d \phi\right)\right\} .
\end{array}
$$

The spatial cross section of $R=0$ has the following induced metric 


$$
d l^{2}=m_{1}^{2} \cosh ^{4 / 3} \gamma\left[d \Omega^{2}+2\left(\frac{L}{\sqrt{2} m_{1} \cosh \gamma} d \tilde{\psi}+\cos \theta d \phi\right)^{2}\right] .
$$

In order for the above metric to be smooth, just as in the vacuum case [2], the following quantization condition must be imposed

$$
m_{1}=\frac{L}{2 \sqrt{2} \cosh \gamma} n_{1}
$$

where $n_{1}$ is an integer. With this condition imposed the metric (20) describes the standard smooth metric on the lens space $L\left(n_{1}, 1\right)$. In this way we obtained the analytical extension of our metric across the surface $R=0$. In completely analogous way one can build the analytical extension of the metric across the surface $R_{2}$ with the following quantization condition

$$
m_{2}=\frac{L}{2 \sqrt{2} \cosh \gamma} n_{2}
$$

where $n_{2}$ is an integer. Therefore the topology of the second horizon is $L\left(n_{2}, 1\right)$.

The asymptotic behavior of the solution is the following

$$
\begin{aligned}
& d s^{2} \simeq\left(1+\frac{2 m \sinh ^{2} \gamma}{R}\right)^{-1 / 3}\left\{-\left(1-\frac{2 m}{R}\right) d t^{2}+\left(1+\frac{2 m \cosh ^{2} \gamma}{R}\right)\left(d R^{2}+R^{2} d \Omega^{2}\right)\right. \\
& \left.+\frac{n^{2} L^{2}}{4}\left(-\frac{2}{R \cosh \gamma} d t+\frac{2 d \psi}{n}+\cos \theta d \phi\right)^{2}\right\} \\
& A_{\mu} d x^{\mu} \simeq-\frac{\sinh \gamma m^{2}}{2 R^{2}} d t+\frac{\sinh \gamma m}{\sqrt{2} R} L d \psi-\cosh \gamma \sinh \gamma m \cos \theta d \phi
\end{aligned}
$$

with $m=\sum_{i} m_{i}$ and $n=\sum_{i} n_{i}$. From the explicit form of the asymptotic metric it is clear that the topology of spatial infinity is $L(n, 1)$.

It is worth noting that in the case of two black holes there is an additional Killing vector $\frac{\partial}{\partial \phi}$ and the techniques based on the notion of interval structure [5] can be applied and they give the same results as the above analysis.

We proceed further with calculating the masses and angular momenta. The mass of each black hole is given by the Komar integral

$$
M_{i}=\frac{-3}{32 \pi} \int_{H_{i}} \star d \xi
$$

where $\star$ is the Hodge duality operator and $\xi$ is the 1 -form corresponding to the timelike Killing vector. The explicit calculation gives the following result 


$$
M_{i}=\frac{3}{2} \pi L m_{i}
$$

The total mass of the multi-black hole configuration is

$$
M=\frac{-3}{32 \pi} \int_{\infty} \star d \xi=\frac{3}{2} \pi L \sum_{i} m_{i}\left(1+\frac{1}{3} \sinh ^{2} \gamma\right) .
$$

The deference $M-\sum_{i} M_{i} \neq 0$ reflects the contribution of the electromagnetic and dilaton fields to the total mass.

The angular momentum of each black hole is defined by the Komar integral

$$
J_{i}^{\psi}=\frac{1}{16 \pi} \int_{H_{i}} \star d \eta
$$

where $\eta$ is 1-form corresponding to the Killing field $\frac{\partial}{\partial \psi}$. The explicit calculation gives

$$
J_{i}^{\psi}=\frac{L^{2}}{\sqrt{2}} \pi m_{i}
$$

The angular velocity of each black hole is $\Omega_{H}=\frac{\sqrt{2}}{L}$ which, combined with the expressions for the mass and angular momentum, results in the following relation

$$
M_{i}=\frac{3}{2} \Omega_{H} J_{i}^{\psi}
$$

For the total angular momentum of the configuration we find

$$
J^{\psi}=\frac{1}{16 \pi} \int_{\infty} \star d \eta=\frac{L^{2}}{\sqrt{2}} \pi \sum_{i} m_{i}=\sum_{i} J_{i}^{\psi} .
$$

Therefore the electromagnetic field gives no contribution to the total angular momentum.

The total electric charge of the configuration is defined by

$$
Q=\frac{1}{4 \pi} \int_{\Sigma} e^{-2 \alpha \varphi} \star F
$$

and the calculations give $Q=0$. This result can be easily seen from the asymptotic behavior $A_{t} \sim \frac{1}{R^{2}}$. The electric charge of each black hole in the configuration is also zero, $Q_{i}=0$. The nontrivial quantity that characterizes the electromagnetic field is the magnetic flux $\Gamma$ through the base space $S_{\infty}^{2}$ of the $S^{1}$-fibration $L(n, 1)$ at infinity, namely 


$$
\Gamma=\frac{1}{4 \pi} \int_{S_{\infty}^{2}} F=\cosh \gamma \sinh \gamma m .
$$

It is also interesting to find the magnetic flux through a portion of the horizon cross sections. It is known however, that in the general case, the magnetic flux lines are expelled from the extremal black holes, more precisely the components of the field strength normal to the horizon vanish [6], [7]. In our case we have

$$
\left.F\right|_{H_{i}}=0
$$

and therefore, within the framework of the present solution, the black hole horizons are superconducting in the sense that they exhibit "Meissner effect" typical for the superconductors. Even more, the electric field also vanishes on the horizons.

We now are at a position to derive a Smarr-like formula giving a relation between the Komar mass and the magnetic flux. For this purpose we consider the 1-form $\chi=i_{\eta} i_{\xi} e^{-2 \alpha \varphi} \star F$ which is closed $d\left(i_{\eta} i_{\xi} e^{-2 \alpha \varphi} \star F\right)=0$ as a consequence of the Killing symmetries and the Maxwell equations. The 1-form $\chi$ is invariant under the Killing symmetries and therefore it can be viewed as defined on the orbit (factor) space spacetime/isometry group. Since the factor space is simply connected [5], there exists a potential $\Psi$ such that $d \Psi=i_{\eta} i_{\xi} e^{-2 \alpha \varphi} \star F$. The explicit expression for $\Psi$ in our case is

$$
\Psi=-\frac{\cosh \gamma \sinh \gamma}{2} \frac{1-H^{-2}}{\cosh ^{2} \gamma-H^{-2} \sinh ^{2} \gamma}+\frac{\tanh \gamma}{2},
$$

where we have fixed the arbitrary constant in the definition of $\Psi$ so that $\Psi$ vanishes on the horizons. Using then the same approach as in [8] it can be shown that the following relation is satisfied

$$
M=\sum_{i} M_{i}+\pi L \Psi(\infty) \Gamma
$$

This is in fact eq.(27) as can be checked.

The ergosurface, defined by $g(\xi, \xi)=0$, exists always since $\left.g(\xi, \xi)\right|_{H_{i}}>0$ and $\left.g(\xi, \xi)\right|_{\infty}<0$ and its topology depends on the point sources configuration just as in the vacuum case.

As a final remark it is worth noting that our preliminary investigations show that there exist solutions describing rotating extremal multi-black hole configurations with selfgravitating electromagnetic field, more general than the solutions presented in [4] and in the present paper. The results will be presented elsewhere.

\section{Acknowledgements}

The partial financial supports from the Bulgarian National Science Fund under Grant DMU-03/6, and by Sofia University Research Fund under Grant 148/2012 are gratefully acknowledged. 


\section{References}

[1] S. Tomizawa and H. Ishihara, Prog. Theor. Phys. Suppl. 189, 7 (2011); arXiv:1104.1468[hep-th]].

[2] K. Matsuno, H. Ishihara, M. Kimura and T. Tatsuoka, Phys. Rev. D86, 044036 (2012); [arXiv:1206.4818v2 [hep-th]].

[3] G. Clement, Gen. Rel. Grav. 18, 861 (1986).

[4] K. Matsuno, H. Ishihara, M. Kimura and T. Tatsuoka, arXiv:1208.5536[hepth]].

[5] S. Hollands and S. Yazadjiev, Commun. Math. Phys. 302, 631 (2011); arXiv:0812.3036 [gr-qc]].

[6] J. Bicak and L. Dvorak, Phys. Rev. D22, 2933 (1980).

[7] A. Chamblin, R. Emparan and G.W. Gibbons, Phys. Rev. D58, 084009 (1998); arXiv:hep-th/9806017.

[8] P. Nedkova and S. Yazadjiev, Phys. Rev. D85, 064021 (2012); arXiv:1112.3326 [hep-th]]. 